\title{
Needs analysis and development of a staff well-being program in a pediatric oncology, hematology, and palliative care services group
}

This article was published in the following Dove Press journal: Journal of Healthcare Leadership

\author{
Penelope J Slater' \\ Rachel M Edwards ${ }^{2}$ \\ 'Oncology Services Group, \\ Queensland Children's Hospital, South \\ Brisbane, QLD, Australia; ${ }^{2}$ Nursing \\ Learning and Workforce Development, \\ Queensland Children's Hospital, South \\ Brisbane, QLD, Australia
}

Purpose: Around 170 multidisciplinary staff of the Oncology Services Group at Queensland Children's Hospital, Brisbane, care for children with oncology, hematology, and palliative care needs from throughout Queensland and northern New South Wales. A series of challenges impacted staff resilience and retention, and strategies were needed to improve staff well-being and enable them to flourish despite the inherent work stressors.

Methods: A needs analysis was conducted using themes from Discovery Interviews with 51 staff, surveys related to "The Work Stressors Scale - Pediatric Oncology" and "The Work Rewards Scale - Pediatric Oncology" completed by 59 staff, and an organizational staff survey responded to by 51 staff.

Results: The needs analysis informed the development of a customized Oncology Staff Well-being Program with a range of strategies aligned to a PERMA framework for flourishing (positive emotion, engagement, relationships, meaning, and accomplishment). Positive emotion areas included education on topics such as well-being, resilience, responding to escalating behaviors, grief and loss, and self-care. Staff attended the available mindfulness sessions, debriefing and counselors on site, developed self-care plans, and followed a well-being Facebook Group. Engagement was supported through exploring character strengths, improving communication, supporting innovation, and addressing frustrations and safety concerns. Relationships within the team were addressed through team building and social events. Meaning of the work was emphasized through sharing family updates and end of treatment celebrations. Accomplishments of staff were acknowledged in newsletters and meetings.

Conclusion: The needs analysis drove a multifaceted approach to staff well-being with the development of strategies which aligned to a framework that would empower staff to flourish at work. Implementation and evaluation are ongoing and will be reported in a subsequent paper.

Keywords: staff well-being, resilience, burnout, vicarious trauma, self-care

\section{Introduction}

Burnout is a complex physiological and psychological experience or response of an individual to stress as it relates to their workplace. Unlike acute stress such as that following trauma, burnout occurs in response to prolonged exposure to stress. ${ }^{1,2}$ The literature describes three distinct characteristics of burnout which include exhaustion, cynicism, and inefficacy which reflect the experience of burnout as seen in the context of human services. ${ }^{2}$ Measurable consequences include emotional exhaustion, negative views, reduced personal accomplishment, and de-personalization. ${ }^{3,4}$ It has been well documented that medical staff ${ }^{-12}$ and nursing staff $f^{6,13-19}$ are heavily impacted, as well as allied health, administrative, and support professions in health care. ${ }^{14,20,21}$
Correspondence: Penelope J Slater Oncology Services Group, Queensland Children's Hospital, 50I Stanley St, South Brisbane, QLD 4I0I, Australia

Tel +6I 730685785

Fax +6I 730684139

Email penny.slater@health.qld.gov.au 
Burnout has been reported by $31 \%$ of cancer workers in Queensland. ${ }^{20}$ High scores in at least one measure of burnout were found in $49 \%$ of radiation oncologists in Australia and New Zealand. ${ }^{9}$ A survey by the Clinical Oncology Society of Australia revealed that $33 \%$ of members with direct patient contact and $27 \%$ with no direct patient contact had high levels of emotional exhaustion. ${ }^{22}$

Those who work in pediatric oncology have specific stressors that further increase their risk of burnout, ${ }^{23,24}$ related to establishing long-term compassionate and caring relationships with children and families whilst they traverse through cancer treatment. However, there are also many rewards from working in this area. ${ }^{21,25-28}$ Health practitioners can undergo a transformation with experience, as they reflect, become more self-aware, and learn to sustain themselves through robust coping mechanisms and self-care strategies. ${ }^{29}$

There are organizational and personal factors that may result in a greater risk of burnout. For example, predictors of burnout in Australian oncology health workers included dissatisfaction with leave arrangements, increased hours of patient contact, and perceived need for communication skill training. ${ }^{22}$ Personal risk factors included age, experience, marital status, single parents, long days, medium to high workload, perceived lack of professional work life quality, and poor self-care. ${ }^{18,24}$ Bullying negatively impacted physical and mental health of nursing staff, ${ }^{30}$ and also resulted in trainees having issues with self-esteem, motivation, job satisfaction, and overall health. ${ }^{31}$

Well-being of health care staff has an influence on their work quality and therefore the health outcomes for patients. For example, poor physician wellness resulted in issues with retention, productivity, efficiency, quality of care, patient compliance and satisfaction, and medical errors. ${ }^{7}$ Physician well-being has been shown to affect their empathy, listening, positive attitude, decision-making, and patient safety. ${ }^{12}$ Linkages between well-being of the workforce and quality and cost of care have also been demonstrated. ${ }^{8}$

Reported mitigating strategies for burnout include mindfulness meditation, reflective writing, supervision, workload control, recognition and reward, supportive teams in a culture of psychological safety, self-care planning, good communication skills, and education on resiliency. ${ }^{8,13,16,29,32,33}$ Diverse strategies that target specific staff needs are required to encourage mental and emotional stability and build resilience in health care professionals. ${ }^{10}$

The Institute for Healthcare Improvement Framework for Improving Joy at Work $^{34}$ recommended that organizations take steps to build well-being and resilience, commencing with asking staff what matters to them and identifying factors that reduce their joy at work. The Framework provided a multifaceted, positive way to facilitate staff well-being and gave direction for the development of the well-being program described in this study.

The Oncology Services Group, Queensland Children's Hospital (QCH), at Children's Health Queensland (CHQ), is a family centered statewide service providing acute and longterm care for patients with malignancy and non-malignant hematological disorders throughout Queensland and northern New South Wales, as well as those receiving palliative care. Associated staff comprise 25 medical, 101 nursing, 32 allied health, and 16 administration staff.

Historically, staff well-being has been addressed within the service in various ways and usually in response to the immediate issues faced by a discipline group or cohort of staff. For example, the administration team in the service overcame some issues in 2010 with targeted strategies around culture, vision and purpose, the provision of information, and improving communication, relationships, and leadership. ${ }^{35}$ Collaborative work undertaken in 2010 for the whole service, based on the Queensland Health Staff Survey, resulted in the development of a Team Charter of Behavior which included practical strategies for staff. Anecdotally, this charter was reasonably successful in some areas of the service where leaders promoted its implementation. The education curriculum for Oncology Services Group has also incorporated well-being content for many years, including topics such as self-care, self-awareness, professional boundaries, courageous conversations, communication skills, surviving organizational change, and providing effective feedback. In addition to these strategies, informal debriefing was provided as well as regular Mortality and Morbidity Meetings, where the clinical, psychosocial, and emotional aspects of complex cases were discussed by the multidisciplinary team.

Following the move from the Royal Children's Hospital to QCH in late 2014, there were significant challenges with settling into the new facility, team workload, public scrutiny, and consumer expectations. Concurrently, there were an increasing number of high acuity and complex patients. This resulted in issues with staff resilience and retention which impacted the team during 2015 and 2016. In order to determine the best way to support staff, a needs analysis was conducted to develop a program with the goal of improving the well-being and resilience of oncology staff, enabling them to cope with the inherent work stressors and to flourish. This paper will report on the needs analysis and will discuss the well-being program which was developed in response to the findings. 


\section{Materials and methods}

Information on staff needs was collected through three processes: the annual organizational survey conducted by the Queensland Government, a stressors and rewards survey, and staff interviews. All three samples were taken from the same staff pool in the Oncology Services Group which consisted of 25 medical, 101 nursing, 16 administration, and 32 research/quality/allied health staff. Both surveys were done anonymously, so the extent of overlap of the samples is unknown. However, the occupational grouping was known and is indicated in the following section.

The Discovery Interviews were performed under approval from the Queensland Health - Central Office Human Research Ethics Committee HREC/10/QHC/51 and written informed consent was obtained from all participants. For the staff surveys, a waiver of ethics review was granted by the CHQ Hospital and Health Service Human Research Ethics Committee for this project as a quality assurance activity conducted within all best practice ethical guidelines with staff only.

\section{Organizational surveys}

The annual Queensland Government wide on-line "Working for Queensland Survey" ran in April/May 2016, and 89,033 surveys were returned. ${ }^{36}$ For the Oncology Services Group, the survey was responded to by 51 staff, including 23 medical, 18 nurses, and seven administration staff. Recruitment was conducted via an email with associated promotional material from the organization, and completion of the survey was voluntary. Informed consent was obtained during the survey process. The results were used as a baseline for the Oncology Staff Well-being Program, and the survey was repeated in August 2017. Reports on the survey were issued through the Queensland Government. ${ }^{36}$ Most data in the survey reports were expressed as $\%$ positive, $\%$ neutral, or $\%$ negative. The measure of $\%$ positive represented the proportion of respondents who expressed a positive opinion in their assessment. Comparisons of the $\%$ positive scores were supplied between areas of the service where an adequate sample size existed. In this way, comparisons could be made between the total Queensland Government results ( $\mathrm{n}=89,033$ ); CHQ results $(\mathrm{n}=2,052)$; the Division of Medicine (where Oncology occurs in the organizational structure) $(\mathrm{n}=278)$; Oncology Services Group ( $\mathrm{n}=51)$, oncology medical group $(\mathrm{n}=23)$; and oncology nursing group $(\mathrm{n}=30)$.

The survey collected information on:

- The individual's employment

- Workplace factors including agency engagement, job empowerment, workload and health, learning and devel- opment, the workgroup, the manager, organizational leadership, organizational fairness, anti-discrimination, and innovation

- Workplace climate including the respondent's job, leadership and engagement, performance and development, fairness and trust, people and relationships, effectiveness and innovation and safety, health and wellness

- Leadership behavior

- Flexible work options

- Domestic and family violence

- Intention to leave the organization

- Bullying and sexual harassment

- The respondent's general view of the organization

- Agency-specific questions, which were around staff wellbeing for $\mathrm{CHQ}$

\section{The Work Stressors Scale and the Work Rewards Scale}

Nursing staff were invited to complete "The Work Stressors Scale - Pediatric Oncology" and "The Work Rewards Scale - Pediatric Oncology" in December 2016 (both copyright 2012, The University of York; All rights reserved). ${ }^{37,38}$ Both surveys were completed by 59 nurses from the Oncology Inpatient Unit $(\mathrm{n}=51)$ and the Oncology Day Unit $(\mathrm{n}=8)$. Recruitment was assisted through advocacy provided by the Nurse Unit Managers of these two areas, voluntary completion, and availability of the survey in hard copy in a common staff area. Written information was provided to potential participants via a broadcast email and completion of the survey implied consent.

Responses for each question as they related to the past 6 months were scored for frequency ("How often have you encountered this situation?") in both the rewards and stressors survey as rarely $=0$, sometimes $=1$, and often $=2$. The Intensity ("How stressful have you found this?" for the stressors survey and "How rewarding has this been for you?" in the rewards survey) was scored in both surveys as not at all $=0$, a little $=1$, and a lot $=2$.

The intensity and frequency scores for each stressor and reward question were summed for the 59 surveys using the above-mentioned scores. For individual surveys with missing data, the total score was rescaled up to the total number of surveys completed. The questions with the highest total intensity score were ranked and examined in relation to the total frequency score. In addition, the percentage of respondents who ranked the question the highest was compared ("a lot" for intensity and "often" for frequency). 
The Work Stressors survey questions had been categorized into three subscales by the designers of the survey: 20 questions related to the child, 20 to the organization, and 20 to the parents. The responses to the questions in each subscale were summed for the 59 surveys or a rescaled total was used to account for missing values. Descriptive statistics were calculated (including the mean and SD of the total responses of the questions within each subscale), and a one-way ANOVA was conducted on the means for each subscale.

\section{Discovery Interviews with staff}

Discovery Interviews were developed in the National Health Service in the UK in $2000^{39}$ as a service improvement tool and patient involvement mechanism in the development of patient-centered services. ${ }^{40-42}$ Generally, the approach uses one-on-one, face-to-face open interview techniques, with some prompting based on key stages of the experience of the service (a spine) ${ }^{8}$

The Discovery Interview methodology was introduced into the Oncology Services Group in 2012 to gain insight into consumer and clinician experience. ${ }^{43}$ Interviewers were trained and one was nominated to be trained in the de-identification and condensing of interview transcripts, relaying those stories to the clinical teams and reporting on service improvement potentials.

For this study, 43 Discovery Interviews were conducted with 51 staff ( 40 individuals and one group of 11 new starter nurses) at all levels of the Oncology Services Group during August and September 2016, including 36 nurses, four educators, six allied health staff, and five administration staff. Twenty-eight of the staff came from the Oncology Inpatient Unit, five from the Oncology Day Unit, and 18 from the directorate and statewide services. Recruitment was assisted with roster adjustment by the Nurse Unit Managers, and emails were sent to all staff to advertise the opportunity to be involved, with the participant information sheet and consent form attached.

The interviews took place in the hospital in an interview room. The interviewee was taken through a participant information sheet and consent form, which was signed, with instructions given about how to revoke an interview from the pool.

The spine used in the staff interviews to guide the conversation had the following topics: being part of cancer services, feeling safe, working with others, solving problems, seeking help and support, receiving help and support, continuing to provide care. Interviewees could start with any of the topics in the spine, as they sparked memories of their experience, or they could work their way through the spine systematically. Interviews were conducted by the Program Manager of the Oncology Services Group who was trained and experienced in the methodology.

Interviews lasted anywhere between 30 minutes and 2 hours, as guided by the interviewee, and were audio-recorded. Interviews were transcribed and de-identified (patient, family, and clinicians).

Qualitative data were analyzed from interviews using an inductive thematic approach. ${ }^{44}$ The Discovery Interview methodology is not designed to provide a representative sample, but to discover insights into the staff experience that cannot be gained in other approaches.

\section{Results Working for Queensland Survey}

The responses from the Oncology Services Group in the 2016 Working for Queensland Survey related to well-being were as follows. Figures given refer to the percentage of positive responses except for the bullying figures. Differences of more than $5 \%$ were highlighted in the reports.

- Overloaded with work (24\% Oncology Services Group total compared with 29\% for total Queensland Government, $29 \%$ CHQ, $17 \%$ oncology medical, $27 \%$ oncology nursing)

- Feel burned out by work (35\% Oncology Services Group total compared with $41 \%$ for total Queensland, $43 \% \mathrm{CHQ}$, $39 \%$ oncology medical, $37 \%$ oncology nursing)

- Work has a negative impact on health $(47 \%$ Oncology Services Group total compared with $45 \%$ for total Queensland, 46\% CHQ, 41\% oncology medical, 45\% oncology nursing)

- Workplace culture supports work/life balance $(58 \%$ Oncology Services Group total compared with $62 \%$ for total Queensland, 60\% CHQ, 57\% oncology medical, $59 \%$ oncology nursing)

- My work/life balance satisfaction (58\% Oncology Services Group total and CHQ, 62\% for total Queensland, $52 \%$ oncology medical, $64 \%$ oncology nursing)

- Bullying and sexual harassment were witnessed by $40 \%$ of Oncology Services Group total staff (27\% total Queensland, 27\% CHQ, 39\% oncology medical, 43\% oncology nursing); 19\% of Oncology Services Group total staff reported being subjected to bullying, (compared with 16\% for total Queensland, 15\% CHQ, 13\% oncology medical). 


\section{The Work Stressors Scale and the Work Rewards Scale}

The top ten stressors and rewards are listed from the highest to the lowest based on total rescaled intensity score, and examined with the percentage of staff who had responded that this had impacted them "a lot" (intensity) and "often" (frequency) (Tables 1 and 2).

Table 3 shows the percentage of each response type by the subscales of child, organization, and parents, indicating that child-related stressors resulted in a higher intensity of response by the staff and also occurred in higher frequency. The mean rescaled total scores for intensity of work-related stress for all questions related to the child was
60.8, organization 52.6, and parents 47.1 (Table 4). These were significantly different (one-way ANOVA $F=4.32, d f$ $=59, P=0.018)$.

\section{Discovery Interviews with staff}

Table 5 shows how the themes from staff interviews were developed into a well-being program that prioritized expressed needs. Finding meaning at work was very important for staff to sustain themselves. They spoke about why they did their work, and how it was a privilege to walk the journey with the families and help them. Their motivation was around making the life of the patient and family as easy as possible in a difficult time.

Table I Work Stressors Scale - Pediatric Oncology results, listing the top ten stressors in terms of total rescaled scores for intensity from the 59 surveys

\begin{tabular}{|c|c|c|c|c|}
\hline Stressors & $\begin{array}{l}\text { Child/ } \\
\text { organization/ } \\
\text { parent }\end{array}$ & $\begin{array}{l}\text { Total } \\
\text { intensity } \\
\text { score }\end{array}$ & $\begin{array}{l}\text { Intensity } \\
\text { \% “a lot” }\end{array}$ & $\begin{array}{l}\text { Frequency } \\
\% \text { "often" }\end{array}$ \\
\hline When a child deteriorates very quicklya & C & 87 & 52.6 & 16.9 \\
\hline Dealing with a lot of deaths in a short space of time ${ }^{a}$ & C & 80 & 52.5 & 28.8 \\
\hline Not having time when a family asks for help ${ }^{a}$ & O & 78 & 42.4 & 23.7 \\
\hline Not being able to do my job to the standard I would like & O & 76 & 46.6 & 13.6 \\
\hline Working with a team member who is not pulling their weight ${ }^{\mathrm{a}}$ & O & 75 & 37.9 & 17.2 \\
\hline Working in an environment where there's lots of stress, sadness, and anxiety ${ }^{a}$ & C & 74 & 28.8 & 67.8 \\
\hline Lots of very sick children on the ward at once ${ }^{a}$ & C & 71 & 34.5 & 51.7 \\
\hline Feeling that patient care is being compromised ${ }^{a}$ & O & 69 & 36.8 & 6.9 \\
\hline Staff not passing on the information I need to do my job & O & 69 & 35.1 & 24.1 \\
\hline Parents not supporting for what I am doing with their child & $P$ & 69 & 30.5 & 10.2 \\
\hline
\end{tabular}

Notes: andicates for comparison, the top ten stressors, measured with the intensity of "a lot”, for Royal Children's Hospital, Melbourne and Monash Children's Hospital. ${ }^{27}$

Table 2 Work Rewards Scale - Pediatric Oncology results, listing the top ten rewards in terms of total rescaled scores for intensity from the 59 surveys

\begin{tabular}{|c|c|c|c|}
\hline Rewards & $\begin{array}{l}\text { Total intensity } \\
\text { score }\end{array}$ & $\begin{array}{l}\text { Intensity } \\
\% \text { “a lot” }\end{array}$ & $\begin{array}{l}\text { Frequency } \\
\% \text { "often" }\end{array}$ \\
\hline Feeling that I have made a difference to a child ${ }^{a}$ & 106 & 77.6 & $4 I .4$ \\
\hline Seeing a child get better ${ }^{\mathrm{a}}$ & 106 & 77.2 & 28.1 \\
\hline Doing something that makes a child feel better ${ }^{\mathrm{a}}$ & 105 & 78.9 & 37.9 \\
\hline Working in a team that is committed to the patients ${ }^{a}$ & 104 & 76.8 & 64.9 \\
\hline The feeling that I am doing my job well & 101 & 78.9 & 47.4 \\
\hline Seeing a patient further down the line and how well they are doing & 101 & 78.9 & 19.3 \\
\hline Knowing that we are providing a good service & 100 & 69.0 & 67.2 \\
\hline Working in a supportive team ${ }^{\mathrm{a}}$ & 99 & 75.4 & 52.6 \\
\hline Seeing children at follow-up when they are well and thriving ${ }^{\mathrm{a}}$ & 99 & 74.1 & 27.6 \\
\hline Being able to get a child to interact with $\mathrm{me}^{\mathrm{a}}$ & 98 & 68.4 & 64.9 \\
\hline
\end{tabular}

Notes: andicates for comparison, the top ten rewards, measured with the intensity of "a lot", for Royal Children's Hospital, Melbourne and Monash Children's Hospital. ${ }^{27}$ 
Table 3 Percentage of responses in stressors survey by subscale

\begin{tabular}{|c|c|c|c|c|c|c|}
\hline \multirow[t]{2}{*}{ Subscale } & \multicolumn{3}{|c|}{$\begin{array}{l}\text { Frequency - how often have you encountered } \\
\text { this situation? }\end{array}$} & \multicolumn{3}{|c|}{ Intensity - how stressful have you found this? } \\
\hline & Rarely & Sometimes & Often & Not at all & A little & A lot \\
\hline Child & 27.6 & 33.6 & 44.9 & 24.1 & 36.3 & 39.5 \\
\hline Organization & 34.7 & 32.0 & 34.4 & 36.7 & 30.2 & 35.8 \\
\hline Parents & 37.6 & 34.5 & 20.7 & 39.2 & 33.6 & 24.6 \\
\hline Total & 100.0 & 100.0 & 100.0 & 100.0 & 100.0 & 100.0 \\
\hline
\end{tabular}

Table 4 Descriptive statistics of rescaled total scores for intensity of work related sources of stress for questions by subscale

\begin{tabular}{|c|c|c|c|c|}
\hline Subscale & $\begin{array}{l}\text { Total scores for questions } \\
\text { from } 59 \text { surveys }\end{array}$ & $\begin{array}{l}\text { Mean total response for questions } \\
\text { in the subscale from } 59 \text { surveys }\end{array}$ & SD & $\begin{array}{l}\text { Range } \\
(\text { min-max) }\end{array}$ \\
\hline Child & 1,215 & 60.8 & 12.3 & $42-87$ \\
\hline Organization & I,052 & 52.6 & 16.7 & 13-78 \\
\hline Parents & 942 & 47.1 & 14.9 & $12-69$ \\
\hline Total & 3,209 & 53.5 & 15.6 & $12-87$ \\
\hline
\end{tabular}

Table 5 Discovery Interview themes and sub-themes, how the well-being program addressed some priorities in the first year, the number of staff who mentioned this area, and mapping to Seligman's (20II) PERMA framework

\begin{tabular}{|c|c|c|c|}
\hline Themes & Sub-themes & No & PERMA \\
\hline \multirow[t]{7}{*}{$\begin{array}{l}\text { Caring for } \\
\text { yourself }\end{array}$} & $\begin{array}{l}\text { - Understand your meaning and purpose in the work - updates from families } \\
\text { communicated, inclusion in family visits, and end of treatment celebrations }\end{array}$ & 24 & M \\
\hline & - Self care - plans developed in workshops and followed up by team leaders. & 26 & $P$ \\
\hline & $\begin{array}{l}\text { Examples of self-care in education sessions, eg, music therapy, exercise, and } \\
\text { mindfulness. Mindfulness practices twice per week in clinical areas. Facebook }\end{array}$ & & \\
\hline & Group promoting self-care, events, and resources. & & \\
\hline & - Understand professional boundaries - education and open discussion & 21 & $\mathrm{P}$ \\
\hline & - Getting support - counselor on site once per week & 17 & $P$ \\
\hline & - Implement reflective practice - supervision investigated & 4 & $\mathrm{E}$ \\
\hline Caring for & - Welcome and support new staff & & $\mathrm{R}$ \\
\hline \multirow[t]{5}{*}{ your team } & - Supportive team environment & 28 & $\mathrm{R}$ \\
\hline & - Share the workload & 4 & $\mathrm{R}$ \\
\hline & - Zero tolerance of poor behavior - presentation from Protective Services & Family behavior 16 & $\mathrm{R}$ \\
\hline & $\begin{array}{l}\text { regarding de-escalation, validation skills training, Team Charter of Behavior } \\
\text { review }\end{array}$ & Staff behavior 9 & \\
\hline & $\begin{array}{l}\text { - Support informal debriefing and team huddles - processes reviewed and } \\
\text { debriefs prioritized }\end{array}$ & 9 & $\mathrm{R}$ \\
\hline Your & - Provide flexible work and access to leave & 6 & $\mathrm{P}$ \\
\hline leaders & - Leaders to check-in and give positive feedback to staff & 5 & A \\
\hline \multirow[t]{3}{*}{ caring } & $\begin{array}{l}\text { - Encourage and support innovations and learning from errors - mentoring of } \\
\text { quality activities }\end{array}$ & 2 & $\mathrm{E}$ \\
\hline & $\begin{array}{l}\text { - Be self-aware and manage stress, role model good behavior, and support staff } \\
\text {-leader training undertaken }\end{array}$ & 9 & $P$ \\
\hline & - Manage workloads - ongoing recruitment & 11 & $\mathrm{R}$ \\
\hline Your & - Acknowledge staff achievements - in meetings and newsletters & 2 & A \\
\hline service/ & - Connect and integrate staff - combined Christmas party, plans for social events & 6 & $\mathrm{R}$ \\
\hline organization & - Addressing design and equipment issues & 6 & $\mathrm{E}$ \\
\hline \multirow[t]{2}{*}{ caring } & $\begin{array}{l}\text { - Put in supportive structures - debriefing process reviewed; education on building } \\
\text { resilience, managing vicarious trauma, coping with critical incidents and grief and } \\
\text { loss; well-being champion role description developed; communication books }\end{array}$ & 31 & $\mathrm{P}$ \\
\hline & $\begin{array}{l}\text { - Provide strategic direction and support for continuous improvement - } \\
\text { mentoring quality activities }\end{array}$ & 4 & $\mathrm{E}$ \\
\hline
\end{tabular}


I feel that it's a privilege to be part of the cancer service mostly because of the consumers that we work with ... that they trust us so much to provide a clinical service ... to tell us how they're feeling and ... trust us to be walking this treatment journey with them ... and I think that's the driving force and motivation why I still ... love coming to work every day regardless of other things.

Staff mentioned the uniqueness of the oncology care they provided in the hospital setting. They saw the families longer, often for years, and got to know them, providing holistic care to the whole family. Many staff were attracted to the specialty because of the high skill set required, with constant learning and problem solving. The complexity of the area, with high risk medications and very sick children, made it a daily challenge for staff, which some saw as positive and others as stressful.

I still like the children and the families and doing that primary care delivery ... It is draining. It's ... high acuity and difficult. And there's a lot that can go wrong. And if it goes wrong it can go very wrong. You do not get to make mistakes, so it's stressful.

Staff had self-care plans of various breadths and effectiveness, tailored to their particular personalities. Mention was made of yoga, running, counseling, healthy eating, staff social events, reading, taking designated breaks during the day, holidays, time with family, sleep, mindfulness, meditation, exercise, walking, quilting, bushwalking, reading books, watching TV, drinking wine, and the use of black humor.

I think people have different mechanisms of processing and different abilities to reflect on the impact of working in this area has on them ... knowing I'm finding this distressing because my heart rate's increasing and I'm feeling a bit overwhelmed ... Just knowing what those triggers actually mean for you.

I think even when people pass away ... it's ... kind of like, I'm not entitled to their grief. I can see the grief that the family's going through ... but I've only known them for a year or two ... It's not mine; it belongs to the family. It belongs to the people in their life. I'm definitely entitled to be sad. Because it's a horrible thing that's happened.

Staff had concerns about how the work was impacting on them and felt it important to improve their awareness of how to address this.

I always worry if one day it will all just bubble up. So you know how you just deal with something... but you do not actually deal with it ... I reckon one day we will all just snap.
Sometimes I had given so much at work that I had noth-

ing left to give at home ... I just am emotionally exhausted, I've got nothing left.

Risk factors mentioned by staff included 12-hour shifts, night shift, getting too attached, and caring for patients that resembled their own children. One interviewee mentioned that coming to work when their own child was sick was very difficult and another that working in the area had made them hypervigilant with their own children. Significant change, such as moving to a new hospital, also impacted on their resiliency.

Change has happened and it's really significant and ... everything just feels a little bit broken ... That has a huge impact on your resiliency and your ability to cope with stress and highly emotional situations.

Some staff had rotated out of oncology, gone on secondments, or long-term leave to have a break from the area. Others found working part-time was protective.

Concerns about professional boundaries of newer staff were mentioned by some, as over-investment could lead to burnout. Individually, staff described how they had learnt that they sometimes needed to give themselves some space and had been supported in the re-allocation of their patients. There was also a fine balance between being open with their emotions and maintaining a sense of control.

I guess for more junior staff, they seem to struggle with

... professional boundaries and knowing when to distance yourself and what you can and cannot do. Because if you're too invested in a family that's when you take it home and it obviously leads to burn out.

Staff reported mostly receiving support from each other. External people were often uncomfortable talking about working with children with cancer, which limited the ability of staff to seek support outside of the hospital. For some staff, this was useful, as it encouraged a complete divide between work and home.

I put my music on and I get in the car as a nurse and ...I think about what happened today, what went well, what didn't go well? ... I do that reflection on the drive home ...I walk in the front door, I think ... I'm a mum now ...It is two lives really.

Some staff spoke about the pressure that new staff put on the team, whilst others recognized that new staff had a vast amount to learn in this clinical specialty and they should be supported to ask questions. One staff member suggested that introductions to new staff should be circulated to the team 
and another suggested a photo book of existing staff as a way to assist newcomers.

Many of the staff felt supported by the team, including the medical staff and senior nurses, although this was not the experience of all. Some senior team members were more approachable and supportive than others and several staff had key people who they approached for help and others that they intentionally avoided. The roles of the Clinical Practice Facilitators, along with the Nurse Educator were an important support.

Staff suggested it was helpful to have a shared responsibility for each other's well-being. This included being aware of colleagues' thresholds and signs when they are overwhelmed or if they were having a bad day, and providing practical support. Staff should ask each other if they were ok, and probe with more specific questions, such as, "Do you need help with that?" or "Where are you up to?" They felt it was important for the team to reflect on the day together. One interviewee, however, warned about the team colluding with each other about a problem, rather than genuinely providing support through critical reflection.

At a time when the service was experiencing staff shortages, many staff were finding the workload overwhelming and commented that people were tired and constantly in crisis mode. Workload had an impact on self-care and team care, including the ability to take leave. Sharing the workload was important, and some staff suggested that the clinically critical tasks should be done as a team.

There are so many more demands on everyone ... we are really focused on what is acute, what is critical ... and everything else has fallen to the wayside. So all of those lovely things that we used to do to take care of each other, we're kind of vaguely aware of them but they're so far down the priority list.

The exposure of the staff to poor behavior varied markedly. Some staff found the team supportive, whereas others described it as a negative hierarchical culture. A few staff were anxious about going to the multidisciplinary team meetings and found them intimidating. One was concerned about staff projecting their stress on others and another raised several examples of improper communication.

Other suggestions by staff to improve the team included better collaboration and staff rotation between clinical areas, professional supervision with reflection, team building with the whole multi-disciplinary team, debriefing, nursing handover of families to include allied health, team problem solving, appointing well-being champions, team social events, undertaking quality activities, improving communication of patient deaths, and normalizing well-being activities, eg, counseling, mindfulness, and self-care.

Staff described several aspects of their work environment that were supportive. Their Nurse Unit Manager listened to their concerns, advocated for the unit's needs, gave positive feedback, and had an open-door policy. They supported a family flexible workplace and allowed staff to learn from critical incidents rather than focusing on blame. They empowered workers to engage in quality activities to improve the service. They proactively supported their staff well-being by allowing them to reallocate patients if they needed and offered counseling support. There were regular check-ins with new staff and a comprehensive education program. Recruitment to address staffing levels was being actioned and succession planning with provision for backfill was managed. Leaders of the professional teams also gave staff the opportunity for debriefing and supervision.

Staff praised the education and professional development opportunities offered by the service. They specifically mentioned education regarding how to talk to distressed and relapsed families, professional boundaries, coping with emotions, grief and loss, conflict management, de-escalation of poor behavior with staff and families, and support for open conversations with staff about poor behavior, some of which was already provided.

Suggestions to improve staff well-being included the opportunity to meet with families who returned for follow-up visits after the end of treatment, attending the bell ringing ceremony at the end of treatment, a communication book in each clinical area with news and thank you cards from families, and feedback regarding how patients were progressing in intensive care (with parent permissions). It was also suggested that the organization could further support staff by having consistent standards regarding the management of families, eg, the allocation of single rooms.

There were some design and equipment issues with the new clinical areas which were frustrating staff. These were prioritized to be addressed where possible, to improve staff engagement. Several staff were also frustrated with the lack of appreciation in the organization of the high dependency and complex nature of oncology, especially in regards to the allocation of beds, infection control measures, and staff resourcing, particularly after hours.

The needs analysis led to the development of a customized Oncology Staff Well-being Program with a range of strategies addressing the needs expressed by staff, which were mapped to Seligman's PERMA framework to ensure there 
were no gaps in the program (positive emotion, engagement, relationships, meaning, and accomplishment). ${ }^{45}$ Positive emotion areas included education on topics such as well-being, resilience, responding to escalating behaviors, grief and loss, and self-care; mindfulness sessions; debriefing; self-care plans; counselors on site; and a Facebook Group. Engagement was supported through exploring character strengths, improving communication, supporting innovation, and addressing frustrations. Relationships within the team were addressed through a team building session and social events. Meaning of the work was emphasized through sharing family updates and end of treatment celebrations. Accomplishments of staff were acknowledged in newsletters and meetings. The implementation and its evaluation will be discussed in greater detail in a subsequent paper.

\section{Discussion}

The needs analysis provided a platform for the development of the Oncology Staff Well-being Program. The Working for Queensland Survey 2016 showed that Oncology staff had poor work/life balance and felt burnt out and overloaded by their work. A greater percentage of oncology staff than average in CHQ had witnessed, and were victims of, bullying. The results were consistent with those from an audit in the Victorian public health sector, where $42 \%$ of workers had witnessed bullying or harassment and $25 \%$ had experienced bullying at work. ${ }^{46}$ Given the survey results, the program focused initially on work/life balance and resilience, with a longer term goal of improving the team culture and behaviors.

The Work Stressors Scale showed that patient (child)related issues were not only more frequently experienced "often" by staff, but they resulted in the highest intensity of stress. The sources of stress appeared consistent with the findings of a Victorian study, ${ }^{27}$ with seven of the ten most frequently endorsed sources of work-related stress being evident in both QCH and Victorian staff samples. Another study examining stressors when working with parents of patients found that the highest stressors came from conflicting views about the treatment, demanding or angry parents, relaying bad news, complex families, and communication challenges. ${ }^{26}$ The first three of these were among the top 20 stressors in this study. Specific stressors on nurses in pediatric bone marrow transplant units in the literature included the time juggling clinical tasks which hindered them providing holistic care for their patients, blurring of professional boundaries, and not being heard by doctors, management, and parents. ${ }^{47}$ If nurses only have time to focus on the clinical tasks to be completed, they are less able to experience the positive impact of being a compassionate presence for families and showing authentic empathy. ${ }^{25}$ Not having time to provide excellent care for patients and excessive workload were stressors for our staff, and in the interviews, the senior staff were also concerned about the professional boundaries of newer staff. Issues with being heard were raised predominantly by the allied health staff.

Identifying these stressors provided opportunities to support staff by decreasing the incidence or impact of those stressors. ${ }^{16,28}$ Although some sources of stress in pediatric oncology are inevitable, the goal of well-being programs should be to train staff to be resilient, as well as prevent stress where possible. ${ }^{27}$ For example, where a nurse is caring for a child who is deteriorating quickly, the leader and team members could provide extra check-ins and opportunities to change patient allocation if necessary. During periods when several deaths have occurred, staff can be supported with access to on-site Employee Assistance Program counselors and group debriefing. The stress of delivering bad news to families may be alleviated by interpersonal communication training and robust self-care strategies. By managing workloads and staffing levels and sharing the work equitably as a team, staff will feel they have time to care for patients to an acceptable standard. Workshops to assess and plan self-care and improve resilience will enable staff to better cope with the stressors.

The identification of the high-intensity rewards from the Work Rewards Scale was an opportunity to increase the frequency of these experiences for staff, ${ }^{28}$ particularly seeing a child get better and seeing them well after treatment. Suggestions included a communication book to allow news from families to be shared with the team, being present at end of treatment celebrations, and sharing thank you notes and cards from families. Inpatient staff, who predominantly experienced the acutely unwell hospitalized children and young people, were given the opportunity to rotate into the day care/outpatients area to care for children who are relatively well during and after treatment.

Six of the ten most frequently endorsed sources of workrelated reward from equivalent staff in Victoria ${ }^{27}$ also occurred in the top ten rewards from $\mathrm{QCH}$, and all of them occurred in the top 15 from QCH. Victorian staff rated the relationship with the parents as an important reward, as well as being appreciated by the family, having a long-term or close relationship with parents, helping families through their entire cancer journey, and doing important things for families. ${ }^{26}$ Likewise, the top 20 rewards for QCH staff included getting thanks from parents and sharing the high points with them. 
The Framework for Improving Joy at Work $^{34}$ recommended that the first step in planning a way forward was to ask staff "What matters to you?" The staff interviewed in the development of this program had quite definite strategies that they used to cope with their work, which included self-care, staying connected with the meaning of their work, getting support from peers or external mentors / counselors, maintaining their professional boundaries, and sometimes being seconded out or taking leave to have a break. In the interviews, staff discussed many areas where they had concerns for their own or their team's well-being, and the ways in which their leaders and the organization could support them.

The needs analysis of the Oncology Staff Well-being Program provided insight into the strategies that were necessary to protect oncology staff well-being, based on those approaches that were already working for the staff, their suggestions for improvements and gaps that existed, and the stresses and rewards they experienced. This was translated to a multifaceted well-being program, with staff able to explore the strategies that worked for them as individuals.

The Framework for Improving Joy at Work ${ }^{34}$ recommended that programs identify and enhance the positive attributes in the workplace. The Oncology Staff Well-being Program has been developed to focus on positive ways to improve well-being and build resilience in the team, such as connecting staff with the meaning and purpose of their work, rather than emphasizing the deficits revealed in the needs analysis. The mapping of the strategies to Seligman's ${ }^{45}$ PERMA structure focused staff on the attributes that help people flourish and gave the opportunity to use online profilers to measure well-being along those dimensions and therefore target appropriate self-care strategies. ${ }^{48}$

\section{Conclusion}

The needs analysis performed using staff interviews and surveys highlighted the unique needs of this staffing group. Whilst there were some broad themes among the needs, the analysis confirmed that well-being is very individualized. This is evidenced in the literature and supported the implementation of a systemic multifaceted approach to staff well-being with strategies that would empower staff to flourish. Staff appreciated that their needs were being listened to and that the leadership was developing ways to improve their support within their workplace. The needs analysis has informed education needs and multifaceted organizational and individual support strategies to improve staff well-being and sustain their resilience. Details of the program and its evaluation will be reported in a subsequent paper.

\section{Acknowledgments}

Gratitude goes to the support provided by the other members of the Oncology Staff Well-being Working Group and those staff that provided services for the program, including Dr Wayne Nicholls, Ashraf Badat, Majella Leahy, Cathy Sullivan, Cathy Henry, Amanda Carter, Maggie James, Katie Hyde, Dr Leigh Donovan, Susan Blair and the staff specialists who supported debriefing. We also thank Claire Morley, Angela Saric, Lana Conic and Michael Aust of the People and Culture team; Liz Crowe, the CHQ Well-being and Resilience Working Group; and our external providers, Marion Bell of Optum, Tere Vaka and Penny Gordon of Penny Gordon and Associates.

\section{Disclosure}

The authors report no conflicts of interest in this work.

\section{References}

1. Schaufeli WB, Leiter MP, Maslach C. Burnout: 35 years of research and practice. Career Develop Int. 2009;14(3):204-220.

2. Maslach C, Schaufeli WB, Leiter MP, Burnout J. Job burnout. Annu Rev Psychol. 2001;52:397-422.

3. Maslach C, Jackson SE. The measurement of experienced burnout. J Organ Behav. 1981;2(2):99-113.

4. Maslach C. Stress and Quality of Working Life: Current Perspectives in Occupational Health. Greenwich: Information Age Publishing; 2006.

5. Kash KM, Holland JC, Breitbart W, et al. Stress and burnout in oncology. Oncology. 2000;14(11):1621-1637.

6. Sabo BM. Compassion fatigue and nursing work: can we accurately capture the consequences of caring work? Int $J$ Nurs Pract. 2006;12(3):136-142.

7. Sherman AC, Edwards D, Simonton S, Mehta P. Caregiver stress and burnout in an oncology unit. Palliat Support Care. 2006;4(1):65-80.

8. Wallace JE, Lemaire JB, Ghali WA. Physician wellness: a missing quality indicator. Lancet. 2009;374(9702):1714-1721.

9. Poulsen MG, Poulsen AA, Khan A, Poulsen EE, Khan SR. Work engagement in cancer workers in Queensland: the flip side of burnout. J Med Imaging Radiat Oncol. 2011;55(4):425-432.

10. Epstein RM, Krasner MS. Physician resilience: what it means, why it matters, and how to promote it. Acad Med. 2013;88(3):301-303.

11. Jones MC, Wells M, Gao C, Cassidy B, Davie J. Work stress and wellbeing in oncology settings: a multidisciplinary study of health care professionals. Psychooncology. 2013;22(1):46-53.

12. Gulati S, Dix D, Klassen A. Demands and rewards of working within multidisciplinary teams in pediatric oncology: the experiences of Canadian health care providers. Qual Rep. 2014;19(36):1-15.

13. Leung J, Rioseco P, Munro P, Stress MP. Stress, satisfaction and burnout amongst Australian and New Zealand radiation oncologists. $J$ Med Imaging Radiat Oncol. 2015;59(1):115-124.

14. Montgomery A, Spânu F, Băban A, Panagopoulou E, Demands J. Job demands, burnout, and engagement among nurses: a multi-level analysis of ORCAB data investigating the moderating effect of teamwork. Burn Res. 2015;2(2-3):71-79.

15. Rushton CH, Batcheller J, Schroeder K, Donohue P. Burnout and resilience among nurses practicing in high-intensity settings. Am J Crit Care. 2015;24(5):412-420.

16. Broom A, Wong WK, Kirby E, et al. A qualitative study of medical oncologists' experiences of their profession and workforce sustainability. PLoS One. 2016;11(11):e0166302. 
17. Kay M, O'Dwyer S, Cooke G, Fergusson L. My Health: A Doctors Wellbeing Survey. Brisbane: Medical Employment and Workforce Planning Unit, Metro South Hospital and Health Service (Queensland Health), 2016. Available from: https://metrosouth.health.qld.gov.au/ research/my-health-report. Accessed September 10, 2018.

18. Wu S, Singh-Carlson S, Odell A, Reynolds G, Su Y. Compassion fatigue, burnout, and compassion satisfaction among oncology nurses in the United States and Canada. Oncol Nurs Forum. 2016;43(4): E161-E169.

19. Hall LH, Johnson J, Heyhoe J, Watt I, Anderson K, O'connor DB. Exploring the impact of primary care physician burnout and well-being on patient care: a focus group study. J Patient Saf. Epub 2017 Nov 14.

20. Mullins N, Mcqueen L. Does compassion fatigue affect nurse educators in practice? Nurs Health. 2017;5(1):18-20.

21. Rizo-Baeza M, Mendiola-Infante SV, Sepehri A, Palazón-Bru A, GilGuillén VF, Cortés-Castell E. Burnout syndrome in nurses working in palliative care units: an analysis of associated factors. $J$ Nurs Manag. 2018;26(1):19-25.

22. Girgis A, Hansen V, Goldstein D. Are Australian oncology health professionals burning out? A view from the trenches. Eur J Cancer. 2009;45(3):393-399.

23. Zander M, Hutton A, King L. Coping and resilience factors in pediatric oncology nurses. J Pediatr Oncol Nurs. 2010;27(2):94-108.

24. Hecktman HM. Stress in pediatric oncology nurses. J Pediatr Oncol Nurs. 2012;29(6):356-361.

25. Sabo BM. Compassionate presence: the meaning of hematopoietic stem cell transplant nursing. Eur J Oncol Nurs. 2011;15(2):103-111.

26. Klassen A, Gulati S, Dix D. Health care providers' perspectives about working with parents of children with cancer: a qualitative study. $J$ Pediatr Oncol Nurs. 2012;29(2):92-97.

27. Bowden MJ, Mukherjee S, Williams LK, Degraves S, Jackson M, Mccarthy MC. Work-related stress and reward: an Australian study of multidisciplinary pediatric oncology healthcare providers. Psychooncology. 2015;24(11):1432-1438.

28. Taylor J, Aldridge J. Exploring the rewards and challenges of paediatric palliative care work: a qualitative study of a multi-disciplinary children's hospice care team. BMC Palliat Care. 2017;16(1):73.

29. Mota Vargas R, Mahtani-Chugani V, Solano Pallero M, et al. The transformation process for palliative care professionals: the metamorphosis, a qualitative research study. Palliat Med. 2016;30(2):161-170.

30. Sauer PA, Mccoy TP. Nurse bullying: impact on nurses' health. West $J$ Nurs Res. 2017;39(12):1533-1546.

31. Mccormack D, Djurkovic N, Casimir G. The workplace bullying of healthcare trainees and its effects. APJHM. 2014;9(1):24-27.

32. Kearney MK, Weininger RB, Vachon ML, Harrison RL, Mount BM. Self-care of physicians caring for patients at the end of life: "Being connected... a key to my survival". JAMA. 2009;301(11):1155-1164.
33. Montgomery AJ. The relationship between leadership and physician well-being: a scoping review. J Healthc Leadersh. 2016;8:71-80.

34. Perlo J, Balik B, Swensen S, Kabcenell A, Landsman J, Feeley D. IHI Framework for Improving Joy in Work. Cambridge: Institute for Healthcare Improvement; 2017.

35. Slater PJ. Hospital administration team development and support in a children's cancer service. Aust Health Rev. 2011;35(4):436-443.

36. Queensland Government [homepage on the Internet]. Brisbane: Working for Queensland Survey; 2018. Available from: https://www.forgov.qld. gov.au/working-queensland-survey. Accessed June 10, 2018.

37. Mukherjee SKM. The work-related stressors and work-related rewards scales for paediatric oncology: the development and validation of new tools for research and clinical practice. In: Plenary paper presented at: 44th Congress of the International Society of Paediatric Oncology; October 5-8, 2012; London, UK.

38. Mukherjee S, Beresford B, Tennant A. Staff burnout in paediatric oncology: new tools to facilitate the development and evaluation of effective interventions. Eur J Cancer Care. 2014;23(4):450-461.

39. Bateson J, Brown GCS, Wilcock PM, Carver J, Machin S. Using patient stories to inspire quality improvement within the modernization agency collaborative programmes. J Clin Nurs. 2003;12:1-9.

40. Wilcock PM, Brown GC, Bateson J, Carver J, Machin S. Using patient stories to inspire quality improvement within the NHS Modernization Agency collaborative programmes. J Clin Nurs. 2003;12(3):422-430.

41. Bridges J, Gray W, Box G, Machin S. Discovery Interviews: a mechanism for user involvement. Int J Older People Nurs. 2008;3(3):206-210.

42. Improvement NHS [webpage on the Internet]. Learning from patient and carer experience. A guide to using Discovery Interviews to improve care. Leicester, UK: NHS Modernisation Agency. 2009. Available from: http://www.improvement.nhs.uk/discoveryinterviews. Accessed August $25,2010$.

43. Slater P, Philpot $\mathrm{S}$. Telling the story of childhood cancer: an evaluation of the Discovery Interview methodology conducted within the Queensland Children's Cancer Centre. Patient Intell. 2016;8:39-46.

44. Braun V, Clarke V. Using thematic analysis in psychology. Qual Res Psychol. 2006;3(2):77-101.

45. Seligman ME. Flourish: A Visionary New Understanding of Happiness and Well-Being. New York: Simon \& Schuster; 2011.

46. Auditor-General V. Bullying and Harassment in the Health Secto. Melbourne: Victorian Government Printer; 2016. Available from: https://www.audit.vic.gov.au/sites/default/files/20160323-Bullying.pdf. Accessed July 13, 2018.

47. Morrison CF, Morris EJ. The practices and meanings of care for nurses working on a pediatric bone marrow transplant unit. J Pediatr Oncol Nurs. 2017;34(3):214-221.

48. Butler J, Kern ML. The PERMA-Profiler: a brief multidimensional measure of flourishing. Int $J$ Wellbeing. 2016;6(3):1-48.
Journal of Healthcare Leadership

\section{Publish your work in this journal}

The Journal of Healthcare Leadership is an international, peer-reviewed, open access journal focusing on leadership for the health profession. The journal is committed to the rapid publication of research focusing on but not limited to: Healthcare policy and law; Theoretical and practical aspects of healthcare delivery; Interactions between healthcare and society and evidence-based practices;
Dovepress

Interdisciplinary decision-making; Philosophical and ethical issues; Hazard management; Research and opinion for health leadership; Leadership assessment. The manuscript management system is completely online and includes a very quick and fair peer-review system. Visit http://www.dovepress.com/ testimonials.php to read real quotes from published authors. 